\title{
Endoarterectomy of Common Carotid Artery in SHR as a Model for Neurosurgical Approach
}

\author{
Kinya Ohtsubo* and Ryoichi Horie**
}

When SHR develop hypertension, accelerated vascular noncollagenous and collagenous protein synthesis (adaptive metabolic change, Yamori, 1974) results in the hypertrophy and hyperplasia of the media (adaptive structural change, Folkow, 1958) and in arteriosclerosis due to the increased collagen. As such a thickened arterial wall can be a suitable experimental model for sclerotic arteries in aged man, vascular systems of aged SHR were utilized as model for microvascular surgery using microsurgical technique (Ohtsubo et al : Jap Heart J 16: 309, 1975).

The most popular surgical therapy for the occlusion of the intracranial arteries at present is the anastomosis between superficial temporal artery and middle cerebral artery (STA-MCA). However, by this technique, only the cortical branches of MCA are perfused and blood supply into the deep perforating arteries can not be expected. As the predilection sites of occlusion in MCA is the $M_{I}$ portion, thromboendoarterectomy directly applied to this $\mathrm{M}_{\mathrm{I}}$ portion appears to be more beneficial therapy after the occlusion of MCA. However, there is no report of successful thromboendoarterectomy at such a small artery, the external diameter of which is 2 to $3 \mathrm{~mm}$.

Therefore, thickened cervical carotid arteries of SHR were used as the model of the $\mathrm{M}_{1}$ portion of MCA and endoarterectomy was attempted to obtain the basic information about sufficient conditions necessary for successful surgical removal of thrombi in such a small artery without causing further obstruction after the operation.

Material and Method:

Cervical carotid arteries of SHR over the age of 1 year were used. The external diameter was about $1.5 \mathrm{~mm}$. The rats were anesthezed by the intraperitoneal injection of Nembutal $(25 \mathrm{mg} / \mathrm{Kg}$ ). Carotid endoarterectomy was performed and continuous suturing was done with 10.0 nylon monofilament $\left(\mathrm{Cron}^{\mathrm{R}}\right)$ under trapping with Scovill clips. The contralateral carotid was ligated. The 3 days after the operation, the carotid artery was extirpated to cofirm the passage and histologically examined.

\section{Results and Discussion:}

In $60 \%$ of the cases of experimental endoarterectomy, the carotid arteries were confirmed to be patent. The factors necessary for successful endoarterectomy were the length of endoarterectomy, the vessel diameter, and the speed of local blood flow. The length of endoarterectomy of the rat carotid artery was 1 to $1.5 \mathrm{~cm}$, and it should be $3 \mathrm{~cm}$ at the $M_{I}$ of human $M C A$, because the diameter of rat carotid

From the Japan Stroke Prevention Center, Izumo; Department of Neurosurgery, Kyoto University,** Kyoto; Sohma Hospital,* Sohma. 
arteries was a half of the $\mathbf{M}_{\mathrm{I}}$, and the speed of blood flow was almost same. The present success in the carotid endoarterectomy in rats indicates the practicability of thromboendoarterectomy at the $\mathrm{M}_{\mathrm{I}}$ of $\mathrm{MCA}$ in humans. 